\title{
Molecular Origin of the Elastic State of Aqueous Hyaluronic Acid
}

\author{
Giulia Giubertoni, ${ }^{\dagger}$ Federica Burla, ${ }^{\dagger}$ Cristina Martinez-Torres, ${ }^{\dagger}$ Biplab Dutta, ${ }^{\dagger}{ }^{\dagger}$ Galja Pletikapic, ${ }^{\dagger}$ \\ Eddie Pelan, ${ }^{\ddagger}$ Yves L. A. Rezus, $^{\dagger}$ Gijsje H. Koenderink, ${ }^{* \dagger}{ }^{\dagger}$ and Huib J. Bakker* ${ }^{* \dagger}$ \\ ${ }^{\dagger}$ AMOLF, Science Park 104, 1098 XG Amsterdam, The Netherlands \\ ${ }^{\ddagger}$ Unilever Research and Development Vlaardingen B.V, Olivier van Noortlaan 120, 3133 AT Vlaardingen, The Netherlands
}

Supporting Information

ABSTRACT: The macroscopic mechanical properties of biological hydrogels are broadly studied and successfully mimicked in synthetic materials, but little is known about the molecular interactions that mediate these properties. Here, we use twodimensional infrared spectroscopy to study the $\mathrm{pH}$-induced gelation of hyaluronic acid, a ubiquitous biopolymer, which undergoes a transition from a viscous to an elastic state in a narrow $\mathrm{pH}$ range around 2.5. We find that the gelation originates from the enhanced formation of strong interchain connections, consisting of a double amide- $\mathrm{COOH}$ hydrogen bond and an $\mathrm{N}-\mathrm{D}-\mathrm{COO}^{-}$hydrogen bond on the adjacent sugars of the hyaluronan disaccharide unit. We confirm the enhanced interchain connectivity in the elastic state by atomic force microscopy imaging.

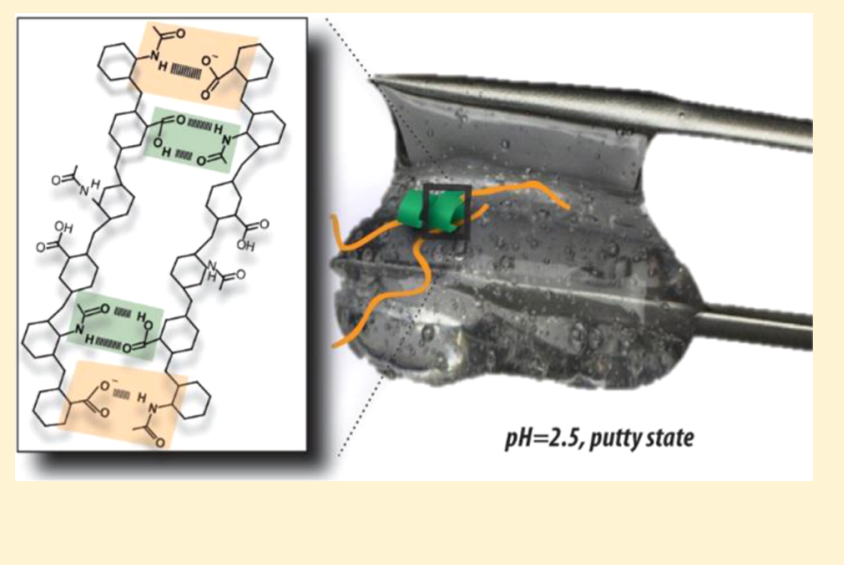

\section{INTRODUCTION}

Hydrogels regulate the mechanical properties of cells and tissues and thereby play an important role in many biological and pathophysiological processes, ranging from stem cell differentiation and the degradation of aging cartilage to wound healing. ${ }^{1-6}$ An important feature of natural hydrogels is their ability to tune their viscoelastic properties in response to environmental stimuli, such as the solution $\mathrm{pH}$, temperature, and salt conditions. Up to now, it has been difficult to connect the macroscopic response of hydrogels to the underlying molecular interactions, e.g., hydrogen-bond and hydrophobic interactions, thus limiting the capability to predict and tune the properties of hydrogels.

One of the few techniques able to track interactions at the molecular scale is two-dimensional infrared spectroscopy (2DIR). ${ }^{7}$ 2D-IR shares with two-dimensional nuclear magnetic resonance the ability to resolve molecular couplings and dynamics, but 2D-IR can resolve these interactions at a much shorter time scale. Molecular interactions such as hydrogen bonds or electrostatic forces usually change on a time scale of picoseconds, and with 2D-IR, snapshots of these interactions can be taken with femtosecond time resolution. ${ }^{8}$

Here, we use 2D-IR to identify the molecular interactions that drive the $\mathrm{pH}$-triggered gelation of hyaluronic acid (HA), a natural polyelectrolyte that has received widespread attention, being one of the main components of the extracellular matrix. It is important for cell-cell communication and also plays an important role in many biological processes, such as joint lubrication, skin hydration, and cell migration, ${ }^{9}$ whereas its dysregulation contributes to cancer development. ${ }^{10}$ Due to its biocompatibility, hyaluronic acid is also widely used as a scaffold for cell culture and drug delivery. ${ }^{11-15}$ Hyaluronic acid is a paradigmatic example of a responsive natural hydrogel: it displays a sharp transition in mechanical behavior at $\mathrm{pH} 2.5$, switching from a viscous state to an elastic gel, denoted as the "putty state". ${ }^{16}$ The mechanism underlying this remarkable liquid-to-gel transition has been investigated for over almost a century with macroscopic techniques that map the dependence of the viscoelastic properties on $\mathrm{pH}$, temperature, and ionic strength. ${ }^{17-19}$ Based on these studies, it has been proposed that at $\mathrm{pH}=2.5$, the net charge of the polyelectrolyte chains may be sufficiently suppressed to enable the formation of interchain hydrogen-bond interactions that would act as crosslinks. $^{20-26}$ This principle was suggested to be applicable to the wider class of polysaccharides, ${ }^{27}$ but there has been no direct evidence for this putative $\mathrm{pH}$-triggered hydrogenbonding mechanism.

\section{EXPERIMENTAL SECTION}

Sample Preparation. The samples were prepared in a glass vial by adding water (or heavy water), $\mathrm{NaCl}(0.15 \mathrm{M})$, and $\mathrm{HCl}$ (or $\mathrm{DCl}$ ) to achieve different molarities ranging from 0 to $80 \mathrm{mM}$ of acid. All of these chemicals were provided by Sigma Aldrich. A specific amount of hyaluronic acid sodium salt in powder form from Streptococcus equi bacteria from Sigma Aldrich (1.5-1.8>1 MDa), or from Lifecore

Received: January 31, 2019

Revised: $\quad$ March 19, 2019

Published: March 19, 2019 
a

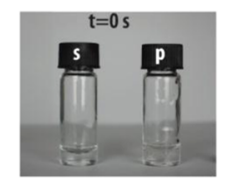

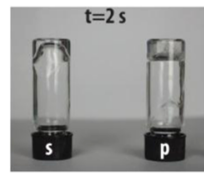
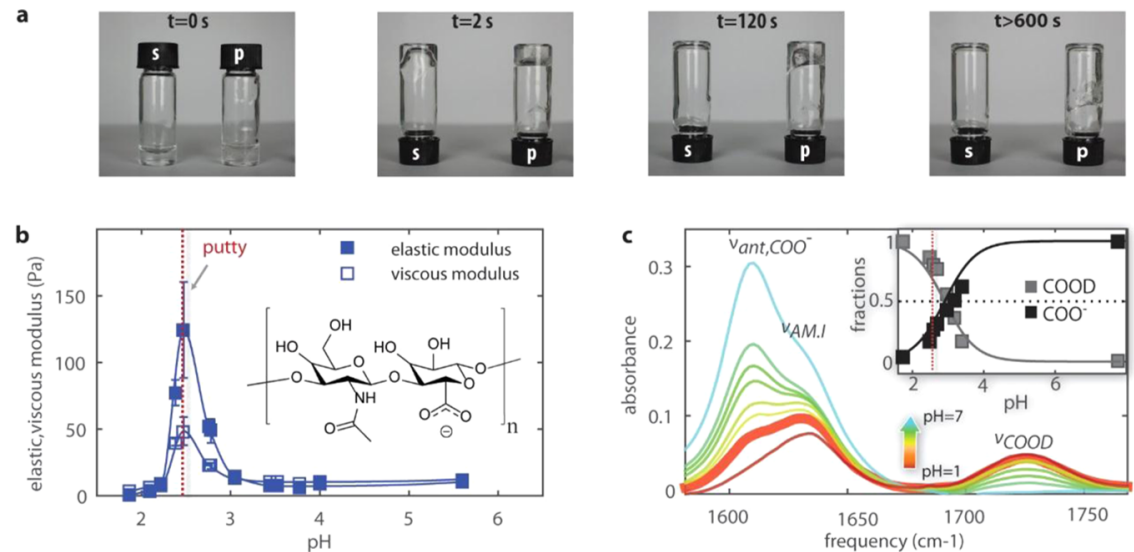

Figure 1. Hyaluronic acid solutions undergo a sol-gel transition in a narrow $\mathrm{pH}$ range. (a) A tube inversion assay shows that hyaluronic acid solutions prepared at $\mathrm{pH} 1.6$ exhibit viscous flow (s), whereas solutions prepared at $\mathrm{pH} 2.5$ form a viscoelastic gel (p) that flows only on time scales beyond $2 \mathrm{~min}$. (b) The $\mathrm{pH}$ dependence of the viscous and elastic modulus of hyaluronic acid in heavy water solutions. We observe a sharp peak in the elastic shear modulus at $\mathrm{pH}$ 2.5. The inset shows that hyaluronic acid is a polymer of disaccharides, themselves composed of $\mathrm{D}$-glucuronic acid and $\mathrm{N}$-acetyl-D-glucosamine. (c) Linear infrared spectra for hyaluronic acid solutions in $\mathrm{D}_{2} \mathrm{O}$ at $\mathrm{pH}$ values ranging between 1.6 and 7 . The infrared spectrum at $\mathrm{pH}=2.5$ is represented by the thick solid line. Between 1550 and $1760 \mathrm{~cm}^{-1}$, we observe three bands: the antisymmetric stretching mode of the carboxylate anion group, $\nu_{\text {ant,COO }}{ }^{-}$, at $1607 \mathrm{~cm}^{-1}$, the amide I vibration, $\nu_{\text {AM.I }}$ at $1633 \mathrm{~cm}^{-1}$, and the carboxylic acid stretching mode, $\nu_{\mathrm{COOD}}$, at $1726 \mathrm{~cm}^{-1}$. The inset shows the fractions of $\mathrm{COO}^{-}$and COOD groups determined from the IR spectra as a function of $\mathrm{pH}$. Thick lines represent the expected fractions based on acid-base equilibrium equations (Supporting Information), assuming a $\mathrm{p} K_{\mathrm{a}}=2.9$. We find good agreement between the measured and expected carboxylic and carboxylate fractions, with about $25 \%$ deprotonation of the carboxyl groups at $\mathrm{pH}=$ 2.5.

Biomedical $\left(M_{\mathrm{w}} \sim 150 \mathrm{kDa}\right)$, was added to the solution to reach a final concentration ranging from 10 to $20 \mathrm{mg} / \mathrm{mL}$. The samples were left to equilibrate at room temperature for at least $24 \mathrm{~h}$ before measuring. Samples were stored for at most 1 week at a temperature of $4{ }^{\circ} \mathrm{C}$, to slow down the hydrolytic degradation process.

Rheology. Rheology measurements were performed with a stress-controlled rheometer (Anton Paar MCR 501), equipped with stainless steel parallel plates with a diameter of $40 \mathrm{~mm}$. The experiments were performed at a gap size of $100 \mu \mathrm{m}$ and at a temperature of $22{ }^{\circ} \mathrm{C}$ set by a Peltier system. Samples left to equilibrate for 1 week to reach homogeneity were loaded on the plate using a spatula. After thermal equilibration, the elastic and viscous shear moduli were probed by performing oscillatory shear measurements at an oscillation frequency of $0.5 \mathrm{~Hz}$ and a strain amplitude of $0.5 \%$, which is well within the linear viscoelastic regime for hyaluronan. The reported results are averages of at least three measurements for each $\mathrm{pH}$. For each sample, the $\mathrm{pH}$ was measured using a $\mathrm{pH}$ meter (Hanna Instruments).

Fourier-Transform Infrared (FTIR). All linear absorption measurements were performed using a Bruker Vertex $80 \mathrm{v}$ FTIR spectrometer, equipped with a liquid-nitrogen-cooled mercury cadmium telluride (MCT) detector. The spectra were recorded under a nitrogen atmosphere at a wavelength resolution of $3 \mathrm{~cm}^{-1}$. For every spectrum, 100 scans were averaged. In all measurements, a standard sample cell with a path length of 100 or $75 \mu \mathrm{m}$ was used. The reported spectra were corrected for the absorption of the solvent background.

2D-IR. In 2D-IR experiments, we performed one-color experiments by pumping and probing around $1680 \mathrm{~cm}^{-1}$ and two-color experiments by pumping at $1680 \mathrm{~cm}^{-1}$ and probing at $1450 \mathrm{~cm}^{-1}$. The home-built setup that we use has been described before. ${ }^{28}$ The excitation was performed with a pair of femtosecond mid-infrared pulses. This excitation pulse pair induced transient absorption changes, which were monitored by a probe pulse that was delayed by a time $T_{\mathrm{w}}$. After transmission through the sample, the probe pulse was sent into an infrared spectrograph and detected with an infrared mercury cadmium telluride (MCT) detector array, thus yielding the transient absorption spectrum as a function of the probe frequency. The dependence of the transient absorption spectrum on the excitation frequency was determined by measuring transient spectra for many different delay times between the two excitation pulses. By Fourier transformation of these spectra, we obtained the dependence of the transient absorption spectrum on the excitation frequency. By plotting the transient absorption spectrum as a function of the excitation and the probing frequency, we obtained a two-dimensional infrared (2D-IR) transient absorption spectrum for each delay time $T_{\mathrm{w}}$.

Atomic Force Microscopy (AFM) Imaging. For AFM measurements, freshly cleaved mica was briefly rinsed with $0.15 \mathrm{M} \mathrm{NaCl}$ before sample deposition. Then, $50 \mu \mathrm{L}$ of 100 $\mathrm{mg} / \mathrm{L}$ HA solution were deposited and left to adsorb for 15 min. The mica was then rinsed with ultrapure water and dried at room temperature. Imaging was performed using tapping mode in air with a TAP150 cantilever on a Veeco Dimension 3100 AFM. The scanning rate was set to $0.5 \mathrm{~Hz}$, with a scan resolution of $512 \times 512$. To retrieve the filament height values, the AFM height images were processed using the open-source software Gwyddion ${ }^{29}$ to correct for a tilt in the image, and the transversal profiles corresponding to the filament cross section were taken. Using a custom script on MATLAB, each profile was corrected for the background noise, and the values of all local maxima were pooled together to obtain a height distribution for each condition tested.

\section{RESULTS}

Rheology and Infrared Spectroscopy. Hyaluronic acid has a relatively simple linear structure and homogeneous composition with repeating disaccharides composed of $\mathrm{N}$ acetyl-glucosamine and glucuronic acid (illustrated in the inset 

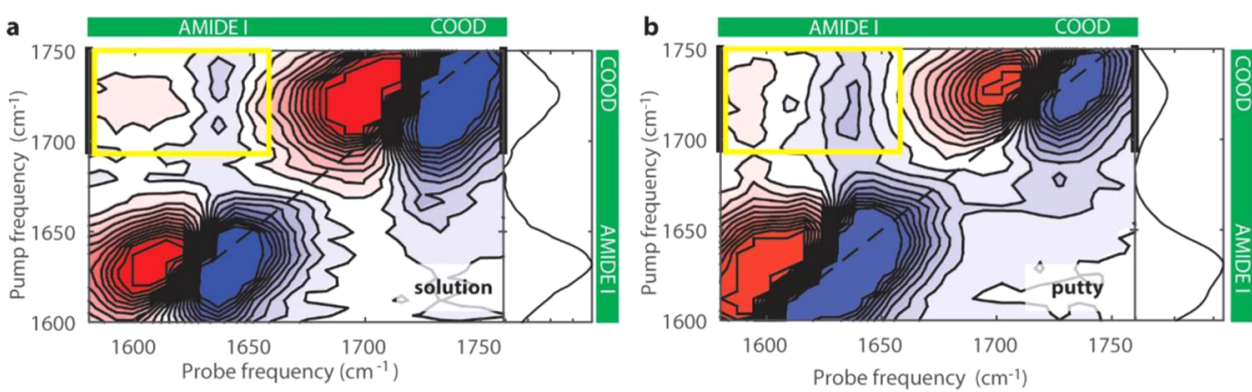

Figure 2. Isotropic two-dimensional infrared spectra of solutions of hyaluronic acid in $\mathrm{D}_{2} \mathrm{O}$ at a concentration of $20 \mathrm{mg} / \mathrm{mL}$ in the spectral region of the carboxylic acid $\left(\nu_{\mathrm{COOD}}\right)$ and the amide I $\left(\nu_{\mathrm{AM} . \mathrm{I}}\right)$ vibrations. 2D-IR spectra $(\mathrm{a})$ for the solution state $(\mathrm{pH}=1.6)$ and $(\mathrm{b})$ for the putty state $(\mathrm{pH}=2.5)$. The corresponding linear spectra are shown on the right-hand side of the $2 \mathrm{D}$-IR spectra. The spectra were collected at a delay time $T_{\mathrm{w}}$ of 1.5 ps. The cross-peaks are indicated by the yellow rectangles and show the presence of vibrational interactions between $\nu_{\mathrm{AM} . \mathrm{I}}$ and $\nu_{\mathrm{COOD}}$. The thick black lines represent the integrated pump region used for the fitting. The pump and probe spectra are centered at $1680 \mathrm{~cm}^{-1}$.

of Figure 1b). The pH-dependent viscoelastic behavior of hyaluronic acid solutions stands out clearly in tube inversion assays, as shown in Figure 1a. Whereas the sample prepared in a solution state ("s") at $\mathrm{pH} 1.6$ readily flows, the sample prepared in the putty state ("p") at $\mathrm{pH} 2.5$ only flows on a time scale of minutes. This large difference in time scales originates from a $\mathrm{pH}$-triggered transition in rheological properties from liquid to gel. To study the $\mathrm{pH}$ dependence of this transition in quantitative detail, we performed rheological experiments on semidilute solutions of high and low molecular weight hyaluronic acid. We studied solutions in heavy water $\left(\mathrm{D}_{2} \mathrm{O}\right)$, since heavy water is used as a solvent in 2D-IR studies of the amide and carbonyl vibrations. 2D-IR cannot be applied to solutions in $\mathrm{H}_{2} \mathrm{O}$, as the amide and carbonyl vibrations of hyaluronic acid absorb in the same infrared frequency region as the bending vibration of $\mathrm{H}_{2} \mathrm{O}$ (between 1600 and $1700 \mathrm{~cm}^{-1}$ ). In all experiments, the $\mathrm{pD}$ values are converted to $\mathrm{pH}$ values ${ }^{30}$ (see Supporting Information (SI) Appendix). Figure $1 \mathrm{~b}$ shows that the elastic and the viscous moduli sharply increase in a narrow range around a $\mathrm{pH}$ of 2.5 , revealing a liquid-gel transition. This transition occurs at a $\mathrm{pH}$ value just below the $\mathrm{p} K_{\mathrm{a}}$, which is about $2.9 .^{12,18,25}$ We find a quantitatively similar $\mathrm{pH}$ dependence of the viscous and elastic moduli for hyaluronan solutions in $\mathrm{H}_{2} \mathrm{O}$ (see Supporting Information Appendix, Figure S2), which shows that the isotope composition of the solvent has a negligible effect on the mechanical properties.

We studied the protonation state of hyaluronan as a function of $\mathrm{pH}$ with linear IR spectroscopy (Figure 1c). The relative intensities of the carboxylic acid and carboxylate anion bands of hyaluronan directly reflect the protonation state of hyaluronan. The antisymmetric vibration of the carboxylate anion, $\nu_{\text {ant,COO }}{ }^{-}$, absorbs at $1607 \mathrm{~cm}^{-1}$, and the amide I band, $\nu_{\text {AM.I }}$ absorbs at $1633 \mathrm{~cm}^{-1}$. The $\mathrm{C}=\mathrm{O}$ vibration of the COOD group, which will be denoted as $\nu_{\mathrm{COOD}}$, absorbs around $1726 \mathrm{~cm}^{-1}$. At $\mathrm{pH}=2.5$, the $\mathrm{COO}^{-}$and COOD groups are present in a ratio of $1: 4$ (inset in Figure 1c), which corresponds to a $\mathrm{p} K_{\mathrm{a}}$ of 2.9 , as reported in the literature. ${ }^{12}$

Two-Dimensional Infrared Spectroscopy. We use 2DIR spectroscopy to study the intermolecular interactions among hyaluronic acid chains that lead to the sol-gel transition. We excite the amide and the carboxyl vibrations with a strong femtosecond infrared pulse pair $(\sim 100 \mathrm{fs}, 4 \mu \mathrm{J}$ per pulse). This excitation induces transient absorption changes that are probed with a weaker $(0.35 \mu \mathrm{J})$ single femtosecond probing pulse that is delayed by a time $T_{\mathrm{w}}$. The excitation and probe pulses are centered at $1680 \mathrm{~cm}^{-1}$ with a bandwidth of $200 \mathrm{~cm}^{-1}$. The excitation and probe spectra thus have sufficient spectral width to cover the $\nu=0$ to $\mathrm{v}=1$ transition (bleach) and the $\nu=1$ to $\mathrm{v}=2$ transition (excited state absorption, or esa) of the $\nu_{\text {ant,COO}}{ }^{-}, \nu_{\mathrm{COOD}}$, and $\nu_{\mathrm{AM} . \mathrm{I}}$ vibrations.

Figure 2a,b compare the 2D-IR spectra measured for the solution $(\mathrm{pH}=1.6)$ and the putty state $(\mathrm{pH}=2.5)$, both for $T_{\mathrm{w}}=1.5$ ps. Bleaching (increased transmission) is indicated in blue, whereas increased absorption (esa) is indicated in red. The bleach of the $\mathrm{v}=0$ to $\mathrm{v}=1$ transition and the esa of $\nu_{\text {AM.I }}$ are centered at 1639 and $1619 \mathrm{~cm}^{-1}$, respectively, along the probe frequency axis, whereas for $\nu_{\mathrm{COOD}}$, these signals are centered at 1731 and $1703 \mathrm{~cm}^{-1}$, respectively (see also Supporting Information Appendix, Table S2). The bleaching and esa of the $\nu_{\text {ant,COO }}{ }^{-}$mode are in the low-frequency wing of the bleaching and esa of the amide I vibration, and lead to an elongation of the bleaching and the induced absorption signals to the lower left corner of the 2D-IR spectrum. We observe additional signals in the off-diagonal regions of the $2 \mathrm{D}$-IR spectrum, indicating that the carboxyl and amide modes are coupled. In the upper pump-frequency region, the cross-peak signal represents the effect of the excitation of the $\nu_{\mathrm{COOD}}$ vibration on the spectrum of the $\nu_{\text {AM.I }}$ mode. In the lower pump-frequency region, the spectral shape of the carboxylic acid vibration is elongated along the pump axis (Figure S7). This effect reduces the visibility of the upward cross-peak, which represents the effect of the excitation of the $\nu_{\text {AM.I }}$ vibration on the spectrum of the $\nu_{\mathrm{COOD}}$ vibration. For this reason, we focus our analysis on the downward cross-peak that represents the effect of the excitation of the carboxylic acid vibration on the $\nu_{\text {AM.I }}$ vibration. The excitation of the $\nu_{\text {COOD }}$ vibration also leads to a cross-peak signal with the amide II mode $\left(\nu_{\mathrm{AM} . \mathrm{II}}\right)$ at $1490 \mathrm{~cm}^{-1}$. This signal results from the excitation of low-frequency modes following the relaxation of the $\nu_{\mathrm{COOD}}$ mode. The excitation of these low-frequency modes affects the absorption spectra of both the $\nu_{\mathrm{COOD}}$ and $\nu_{\mathrm{AM} \text {.II }}$ modes (see SI Appendix, Figure S6a,b).

To learn more about the mechanism that underlies the cross-peak signals of the amide I modes following excitation of the COOD vibration, we compare the dependence on time delay for the solution and the putty states (Figure 3a). We normalized the transients to the bleaching signal of the carboxylic acid vibration at early time delay $(\sim 0.2 \mathrm{ps})$. From $\sim 1.5$ ps on, the decay of the transients is quite similar to that of the $\nu_{\mathrm{COOD}}-\nu_{\mathrm{AM} . I I}$ cross-peak, showing a time constant of $\sim 3$ 


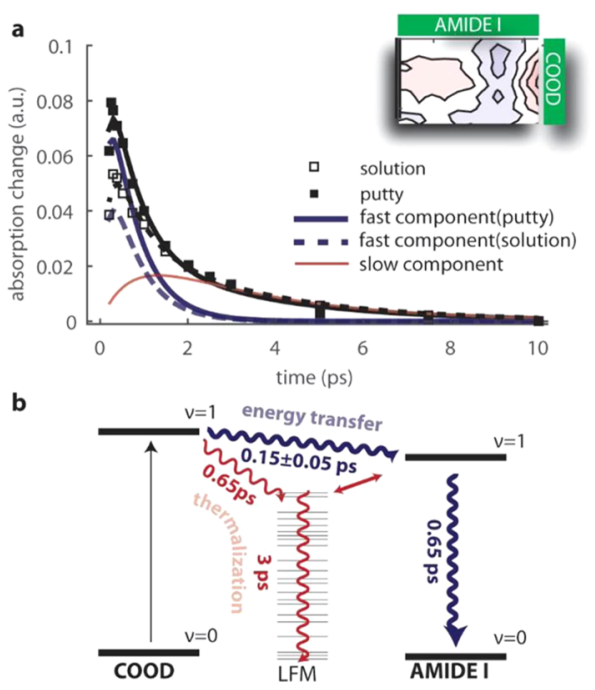

Figure 3. a) Decay traces of the cross-peak in the $\nu_{\text {AM.I }}$ vibrational region for hyaluronic acid at a concentration of $20 \mathrm{mg} / \mathrm{mL}$ in the solution and putty states. The decay traces are normalized to the carboxylic acid intensity at early time delay. The solid and dashed black lines are fits to the relaxation model (described in the Supporting Information), for the putty and solution states, respectively. The blue lines represent the fast component: thick and dashed lines correspond, respectively, to the putty and solution states. The initial amplitude of the fast component (indicated with $c_{\text {ent }}$ ) is nearly two times larger in the putty state $\left(c_{\text {ent }}=0.13 \pm 0.01\right)$ than in the solution state $\left(c_{\text {ent }}=0.077 \pm 0.005\right)$. The red line represents the contribution of the slow component, whose initial amplitude $c_{\text {th }}$ is similar for the putty and solution states $\left(c_{\mathrm{th}}^{\text {putty }} \approx c_{\mathrm{th}}{ }^{\text {sol. }}=0.024 \pm\right.$ $0.004)$. Values reported are the mean and standard deviation obtained by averaging over three different independent measurements. (b) Schematic of the relaxation model describing the cross-peak dynamics. Red and blue lines refer, respectively, to the slow component (due to thermalization) and fast component (due to energy transfer) of the cross-peak dynamics. The value shown is the average with standard deviation for six independent measurements.

ps. However, the transients of the $\nu_{\mathrm{COOD}}-\nu_{\mathrm{AM} \text {.I }}$ cross-peak signals have their maximum at $0.3-0.5 \mathrm{ps}$, a much earlier delay time than the one observed for the $\nu_{\mathrm{COOD}}-\nu_{\mathrm{AM} . I I}$ cross-peak $(\sim 1-1.5 \mathrm{ps})$. In addition, the transients of the $\nu_{\mathrm{COOD}}-\nu_{\mathrm{AM} . \mathrm{I}}$ cross-peak show an additional faster decay component with a time constant of $\sim 0.6 \mathrm{ps}$. Interestingly, the transient of the $\nu_{\mathrm{COOD}}-\nu_{\mathrm{AM} . \mathrm{I}}$ cross-peaks has a much higher intensity at early delay times for the putty state than for the solution state. This observation, together with the dynamics observed for the $\nu_{\mathrm{COOD}}-\nu_{\mathrm{AM} \text {.II }}$ cross-peak, indicates that the $\nu_{\mathrm{COOD}}-\nu_{\mathrm{AM} . \mathrm{I}}$ crosspeak signal contains contributions from two distinct mechanisms: (1) energy transfer from the excited COOD vibration to the amide I vibration; (2) anharmonic coupling to lowfrequency modes that affect the vibrational spectrum of the amide I mode. The relaxation model corresponding to these two mechanisms is illustrated in Figure $3 \mathrm{~b}$.

The cross-peak signal arising from the energy transfer rises with a time constant $T_{\text {ent }}$, representing the time scale of energy transfer from the excited COOD vibration to the accepting amide I vibration, and decays with the vibrational lifetime, $T_{1 \text { AM.I }}$ of the amide I vibration. This direct energy transfer does not occur for the amide II mode because there is a rather large energy difference between the $\nu_{\text {COOD }}$ and $\nu_{\text {AM.II }}$ vibrations. We extract the value of $T_{1 \mathrm{AM} \text {.I }}$ from the diagonal signal of the amide I mode in the $2 \mathrm{D}$-IR spectrum. We find that $T_{1 \mathrm{AM} . \mathrm{I}}$ is comparable to the vibrational lifetime of the COOD vibration: $T_{1 \mathrm{AM} . \mathrm{I}}=0.65 \pm 0.1 \mathrm{ps}$. The time constants of the second mechanism involving anharmonic coupling to low-frequency modes can be extracted from a fit to the transient of the $\nu_{\mathrm{COOD}}-\nu_{\mathrm{AM} \text {.II }}$ cross-peak (SI Appendix, Figure $\mathrm{S} 6 \mathrm{~b})$. We thus fit the transients of the $\nu_{\mathrm{COOD}}-\nu_{\mathrm{AM} . \mathrm{I}}$ cross-peak to the following expression containing the lifetimes of the COOD vibration, $T_{1 \mathrm{COOD}}$, the amide $\mathrm{I}$ vibration, $T_{1 \mathrm{AM} . \mathrm{I}}$, and the low-frequency-modes, $T_{1 \mathrm{LFM}}$

$$
\begin{aligned}
\frac{\Delta \alpha_{\mathrm{CP}}(t)}{\Delta \alpha_{\mathrm{COOD}}(t)}= & c_{\text {anh }}\left(\frac{T_{1 \mathrm{LFM}}}{T_{1 \mathrm{COOD}}-T_{1 \mathrm{LFM}}}\right)\left(\mathrm{e}^{-t / T_{1 \mathrm{COOD}}}\right. \\
& \left.-\mathrm{e}^{-t / T_{1 \mathrm{LFM}}}\right)+c_{\text {ent }}\left(\frac{T_{1 \text { AM.I }}}{T_{\text {ent }}-T_{1 \text { AM.I }}}\right) \\
& \left(\mathrm{e}^{-t / T_{\text {ent }}}-\mathrm{e}^{-t / T_{1 \text { AM.I }}}\right)
\end{aligned}
$$

Here, $\frac{\Delta \alpha_{\mathrm{CP}}(t)}{\Delta \alpha_{\mathrm{COOD}}(\bar{t})}$ is the intensity of the cross-peak normalized to the intensity of the $\nu_{\mathrm{COOD}}$ vibration at early time delay, $\bar{t}=200$ fs. The parameters $c_{\text {anh }}$ and $c_{\text {ent }}$ represent the cross-peak amplitudes associated with the anharmonic coupling and direct energy transfer mechanisms, respectively. $T_{\text {ent }} c_{\text {anh }}$, and $c_{\text {ent }}$ are the only free parameters. As shown in Figure $3 a, c_{\text {ent }}$ is nearly 2fold larger for the putty state than for the solution state, whereas $c_{\mathrm{anh}}$ is comparable for the two states. For the time constant $T_{\text {ent }}$ we obtain a value of $150 \pm 50 \mathrm{fs}$. The energy transfer to the amide I mode constitutes an additional channel for vibrational relaxation of the COOD mode. The fact that we do not observe a significant difference in $T_{1 \text { COOD }}$ between the putty and the solution state indicates that this energy transfer process occurs only for a minor fraction of the COOD groups. From the values of $c_{\text {ent }}$, we estimate this fraction to be $13 \pm 2 \%$ for the putty state and $7 \pm 2 \%$ for the solution state. The time constant $T_{\text {ent }}$ also does not change upon the formation of the putty state, which shows that the efficiency of the energy transfer remains the same. Hence, upon formation of the putty state only the number of COOD groups showing energy transfer increases.

The ultrafast energy transfer between the $\mathrm{C}=\mathrm{O}$ vibration of the COOD group and the amide I vibration of the amide group can be well explained from the presence of a hydrogen bond between the COOD and the amide groups. ${ }^{31}$ To get more information on the nature of this hydrogen bond, we compared the intensity of the $\nu_{\mathrm{COOD}}-\nu_{\mathrm{AM} . I}$ cross-peak signal for polarizations of the probing pulse parallel and perpendicular to the polarization of the excitation pulse. The ratio of these two signals reveals the relative orientation of the transition dipole moments of the excited and probed vibrations that form the cross-peak signal. ${ }^{32}$ From the ratio of the signals at $T_{\mathrm{w}}$ between 0.3 and 1 ps (where the energy transfer mechanism is predominant), we find that the angle between the transition dipoles of $\nu_{\mathrm{COOD}}$ and $\nu_{\mathrm{AM} . \mathrm{I}}$ is $\sim 20^{\circ}$ (SI Appendix, Figure S9). As the transition dipole moment of $\nu_{\text {AM.I }}$ is at an angle of $20^{\circ}$ relative to the $\mathrm{C}=\mathrm{O}$ bond of the amide group, ${ }^{33}$ we conclude that the hydrogen-bond configuration involves a parallel (or anti-parallel) alignment between the $\mathrm{C}=\mathrm{O}$ groups of the carboxylic acid and amide, forming two closely spaced intermolecular hydrogen bonds between the amide and carboxylic acid groups, i.e., $\mathrm{C}=\mathrm{O}-\mathrm{OD} \cdots \mathrm{O}=\mathrm{C}-\mathrm{ND}$ and $-\mathrm{N}-\mathrm{D} \cdots \mathrm{O}=\mathrm{C}-\mathrm{OD} \cdots \mathrm{O}=\mathrm{C}-$. This geometry, in which the amide and carboxylic acid groups act both as the hydrogenbond donor and acceptor stabilizing the hydrogen-bonded 
structure formed by these two molecular groups, constitutes a strong interchain connection. We conclude that the putty state at $\mathrm{pH}=2.5$ contains a 2 -fold higher density of these strong double hydrogen bonds than the solution state at $\mathrm{pH}=1.6$.

To study the role of the $\mathrm{COO}^{-}$groups in the formation of the putty state, we also measure $2 \mathrm{D}$-IR spectra where we excite and probe the $\nu_{\text {ant,COO}}{ }^{-}$and the $\nu_{\text {AM.I }}$ vibrations. Since the absorption bands of these two vibrations overlap, we subtract the parallel signal from three times the perpendicular signal. In the resulting difference spectrum, the diagonal peaks are strongly suppressed since the corresponding parallel signal is $\sim 3$ times larger than the perpendicular signal. This procedure is thus ideally suited to reveal the presence of cross-peak signals of vibrations that are differently oriented. This strategy has been extensively used in 2D-IR experiments on proteins to resolve vibrational couplings. ${ }^{34}$ Figure 4 a reports the resulting

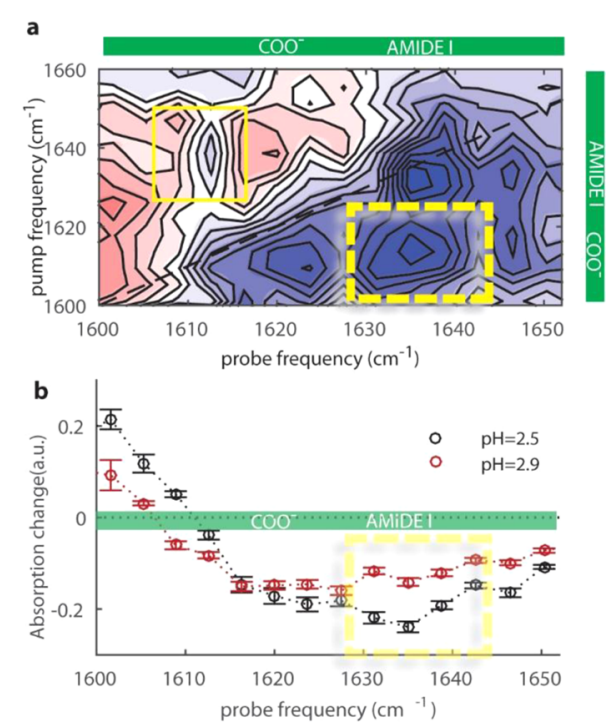

Figure 4. (a) Difference 2D-IR spectrum for a solution of hyaluronic acid at $\mathrm{pH} 2.5$ at a concentration of $20 \mathrm{mg} / \mathrm{mL}$. The difference $2 \mathrm{D}-\mathrm{IR}$ spectrum was obtained by subtracting the parallel signal from three times the perpendicular signal. The yellow rectangles indicate the cross-peak signals of the amide I and $\mathrm{COO}^{-}$modes. (b) Transient absorption spectra obtained by averaging over the pump-frequency region between 1600 and $1620 \mathrm{~cm}^{-1}$ at $\mathrm{pH} 2.5$ and 2.9, and normalized with respect to the pure antisymmetric stretching signal of the carboxylate anion group (Figures S10 and S11).

difference $2 \mathrm{D}$-IR spectrum at $\mathrm{pH}=2.5$ (putty state). On the diagonal, we observe some residual signal due to orientational relaxation of the $\mathrm{COO}^{-}$and amide molecular groups. ${ }^{35-37}$ In addition, we also see clear off-diagonal signals (indicated by the yellow rectangles). In Figure $4 \mathrm{~b}$, we plot the transient absorption spectrum at $\mathrm{pH}=2.5$ (black circles) obtained from Figure $4 \mathrm{a}$ by averaging the signals with pump frequencies between 1600 and $1620 \mathrm{~cm}^{-1}$, corresponding to the excitation of the $\nu_{\text {ant,COO}}{ }^{-}$vibration. The resulting spectrum is compared with the transient absorption spectrum at $\mathrm{pH}=2.9$ that is obtained using the same procedure (SI Appendix, Figure S12). The transient absorption spectrum at $\mathrm{pH} 2.5$ clearly shows an enhanced absorption signal around the amide I vibrational frequency, which implies that the excitation of the $\nu_{\text {ant,COO}}{ }^{-}$ vibration yields an enhanced response of the $\nu_{\mathrm{AM} \text {.I }}$ vibration at $\mathrm{pH}=2.5$. This means that in the putty state $(\mathrm{pH}=2.5)$, the interaction between the $\mathrm{COO}^{-}$and amide molecular groups is enhanced. A similar enhancement is observed for the other cross-peak signal, i.e., exciting the amide I vibration and probing the $\mathrm{COO}^{-}$mode (SI Appendix, Figure S11f). Hence, our results show that the formation of single $\mathrm{N}-\mathrm{D}-\mathrm{COO}^{-}$ hydrogen bonds is enhanced in the putty state, in addition to the enhanced presence of double amide- $\mathrm{COOH}$ hydrogen bonds in this state.

Atomic Force Spectroscopy (AFM). We also perform atomic force microscopy (AFM) imaging of hyaluronic acid solutions at different $\mathrm{pH}$ values to investigate to what extent the additional hydrogen bonds in the putty state are formed within or between hyaluronic acid chains (Figure 5a,b). At $\mathrm{pH}$

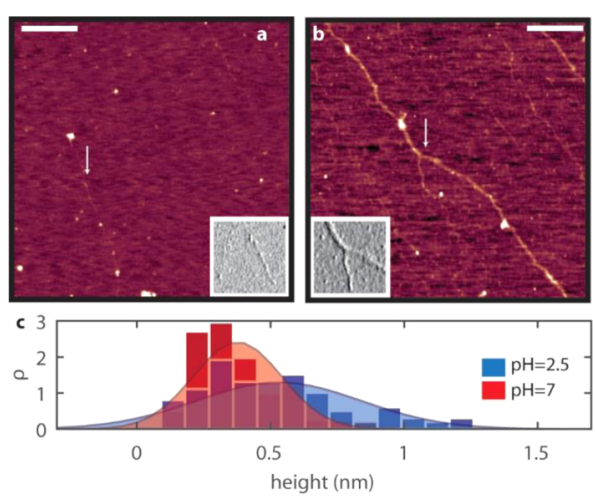

Figure 5. On the top, AFM height images of HA filaments at $\mathrm{pH} 7$ and 2.5, respectively, color coded from $0 \mathrm{~nm}$ (dark red) to $1.2 \mathrm{~nm}$ (white). The insets show amplitude images of smaller areas of $500 \times$ $500 \mathrm{~nm}^{2}$, indicated by the arrows. Scale bar: $500 \mathrm{~nm}$. On the bottom, filament height distributions with Gaussian fits.

$=7$, we mainly observe pearl-necklace conformations: thin chains alternating with thicker globular configurations. The globular portions are likely due to the low affinity for mica. ${ }^{38}$ At $\mathrm{pH}=2.5$, the AFM images clearly show a much larger density of thick filaments than at $\mathrm{pH}=7$, indicative of interchain interactions. To quantify the extent of lateral association of hyaluronan polymers, we measured the heights of the filaments at $\mathrm{pH}=7$ and 2.5 (Figure 5c). At neutral $\mathrm{pH}$, we find a Gaussian height distribution centered at an average filament height $h_{0}=0.37 \mathrm{~nm}$. This value is consistent with the expected thickness of a single hyaluronan chain. ${ }^{38}$ At $\mathrm{pH}=2.5$, we find a much broader distribution, and $h_{0}=0.53 \mathrm{~nm}$, meaning that the filaments are thicker. Since the length of the filaments at $\mathrm{pH}=2.5$ is not changed compared with that at $\mathrm{pH}$ $=7$, we conclude that this thickening is mainly due to enhanced interchain interactions that laterally associate the strands.

\section{DISCUSSION}

The two-dimensional infrared (2D-IR) spectroscopy measurements show that the remarkable $\mathrm{pH}$-triggered gelation of hyaluronic acid at $\mathrm{pH} 2.5$ involves the enhanced formation of double hydrogen bonds between carboxylic acid and amide groups, and of strong single hydrogen bonds between a carboxylate anion (which is a stronger hydrogen-bond acceptor) and amide groups. The enhanced formation of hydrogen bonds is intimately related and even enabled by changes in the structure and electrostatic interactions of the hyaluronan polymer chains.

An important question is why the gelation occurs only in a narrow $\mathrm{pH}$ range. In particular, one may wonder why the 
gelation does not occur for a larger $\mathrm{pH}$ range below 2.5, as at lower $\mathrm{pH}$, there will be even more carboxylic acid groups and thus potentially even more strong double hydrogen bonds with the amide groups located on a different chain.

The answer to this question can be found in the special structure of the hyaluronic acid disaccharide unit, which contains a carboxylic acid group and an amide group on neighboring sugar units (see the inset of Figure $1 \mathrm{~b}$ ). The formation of a double amide-carboxylic acid hydrogen-bond structure involves a quite strict local positioning of the hyaluronic acid chains, which prevents the formation of a second double hydrogen-bond structure at the adjacent saccharide units. Hence, the following mechanistic picture arises (Figure 6): at $\mathrm{pH}=2.5$, the partial deprotonation of the

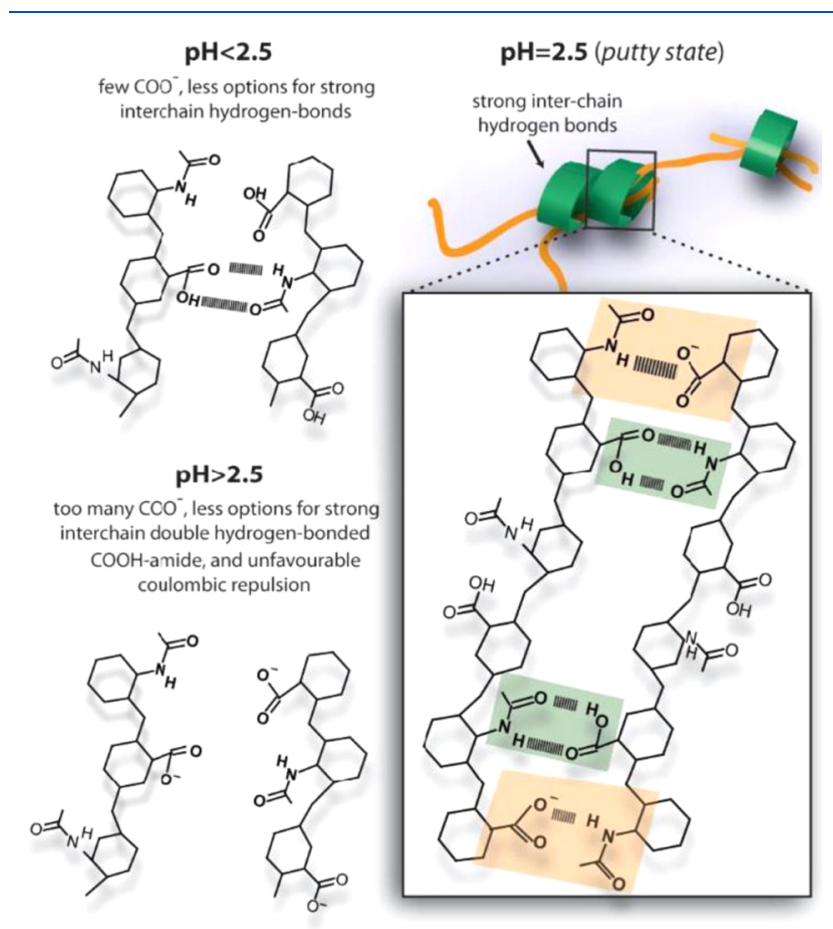

Figure 6. Schematic images showing the transition from a weakly hydrogen-bonded network of hyaluronan at $\mathrm{pH}<2.5$ (top left) to a strongly hydrogen-bonded network at $\mathrm{pH}=2.5$ (right), to isolated single chains at $\mathrm{pH}>2.5$ (bottom left). At $\mathrm{pH}=2.5$, the balance between hydrogen-bond and Coulomb repulsion interactions is optimal, and allows the formation of hydrogen bonds between the carboxyl acid and amide groups observed as the fast time component in the cross-peak dynamics in 2D-IR. Green shaded squares indicate the formation of hydrogen bonds between amide and protonated carboxyl groups, whereas orange shaded squares depict the formation of hydrogen bonds between amide and deprotonated carboxyl groups.

carboxylic acid groups allows for a high probability of forming a strong double amide-COOH hydrogen bond (green box) and an adjacent strong $\mathrm{N}-\mathrm{D}-\mathrm{COO}^{-}$hydrogen bond (orange box), together forming a very strong interchain connection. In the single $\mathrm{N}-\mathrm{D}-\mathrm{COO}^{-}$hydrogen bond, the $\mathrm{N}-\mathrm{D}$ of an amide group acts as the hydrogen bond donor and the $\mathrm{COO}^{-}$ anion group as the hydrogen-bond acceptor. For a sufficient length and concentration of the hyaluronic acid chains, this enhanced hydrogen-bond formation will yield a long-range connected network that behaves as an elastic solid, the putty state. This state is not formed at lower $\mathrm{pH}$, because at these $\mathrm{pH}$ values, very few carboxylic acid groups are deprotonated and the strong double amide- $\mathrm{COOH}$ hydrogen bond can only be combined with a relatively weak single $\mathrm{N}-\mathrm{D}-\mathrm{COOH}$ or single $\mathrm{COOH}$-amide hydrogen bond on the neighboring sugar units, thus forming a weaker interchain connection. As a result, fewer interchain connections are formed (ca. 2 -fold less at $\mathrm{pH}=1.6$ ), and the overall connectivity is too low to form an elastic gel. Also, at $\mathrm{pH}$ values above 2.5 , the putty state is not formed because a large fraction of the carboxylic acid groups is deprotonated, which implies that no double amide- $\mathrm{COOH}$ hydrogen bonds can be formed. In addition, Coulomb repulsion will prevent the formation of $\mathrm{N}-\mathrm{D} \cdots-\mathrm{O}-\mathrm{C}$ hydrogen bonds on neighboring sugar units. As a result, the density of interchain connections is again too low to allow for gel formation.

\section{CONCLUSIONS}

In summary, we studied the molecular origin of the putty state of the biologically important polysaccharide hyaluronic acid, which undergoes a sol-gel transformation only in a narrow range around $\mathrm{pH}=2.5$. Using two-dimensional infrared (2DIR) spectroscopy, we find that this remarkable transition is accompanied by the enhanced formation of strong interchain connections that consist of a strong double amide- $\mathrm{COOH}$ hydrogen bond (green box in Figure 6) and a strong N-D$\mathrm{COO}^{-}$hydrogen bond (orange box in Figure 6) on the adjacent sugar groups of the hyaluronan disaccharide unit. These strong collective interchain connections can only form when the carboxylic acid groups are partially deprotonated, which explains why the putty state is observed only in a narrow $\mathrm{pH}$ range. The enhanced interchain connectivity in the putty state is confirmed by AFM measurements that reveal the association of hyaluronan into thick strands in this state.

This study illustrates that the combination of 2D-IR spectroscopy, AFM, and rheology constitutes a unique tool to identify the molecular mechanisms by which hydrogels respond to environmental stimuli. This combined multiscale approach has great potential to investigate the molecular origin of the biological functionalities of other natural polyelectrolytes and to assist in the rational design of supramolecular biomaterials with life-like functionalities. ${ }^{14}$

\section{ASSOCIATED CONTENT}

\section{S Supporting Information}

The Supporting Information is available free of charge on the ACS Publications website at DOI: 10.1021/acs.jpcb.9b00982.

Data analysis methods; hyaluronic acid gel pictures; rheology experiments in water; linear infrared spectra; anisotropy data; 2D-IR spectra; AFM measurements (PDF)

\section{AUTHOR INFORMATION}

\section{Corresponding Authors}

*E-mail: G.Koenderink@amolf.nl (G.H.K.).

*E-mail: H.Bakker@amolf.nl (H.J.B.).

\section{ORCID}

Giulia Giubertoni: 0000-0002-3417-4987

Biplab Dutta: 0000-0001-8896-2690

Gijsje H. Koenderink: 0000-0002-7823-8807

Huib J. Bakker: 0000-0003-1564-5314

Notes

The authors declare no competing financial interest. 


\section{ACKNOWLEDGMENTS}

This work is part of the industrial partnership programme Hybrid Soft Materials that is carried out under an agreement between Unilever Research and the Netherlands Organisation for Scientific Research (NWO). The authors wish to thank Lukas Helmbrecht for making the photographs.

\section{REFERENCES}

(1) Chaudhuri, O.; Gu, L.; Klumpers, D.; Darnell, M.; Bencherif, S. A.; Weaver, J. C.; Huebsch, N.; Lee, H.; Lippens, E.; Duda, G. N.; et al. Hydrogels with Tunable Stress Relaxation Regulate Stem Cell Fate and Activity. Nat. Mater. 2016, 15, 326-334.

(2) Engler, A. J.; Sen, S.; Sweeney, H. L.; Discher, D. E. Matrix Elasticity Directs Stem Cell Lineage Specification. Cell 2006, 126, 677-689.

(3) Vining, K. H.; Mooney, D. J. Mechanical Forces Direct Stem Cell Behaviour in Development and Regeneration. Nat. Rev. Mol. Cell Biol. 2017, 728-742.

(4) Lee, H.; Gu, L.; Mooney, D. J.; Levenston, M. E.; Chaudhuri, O. Mechanical Confinement Regulates Cartilage Matrix Formation by Chondrocytes. Nat. Mater. 2017, 16, 1243-1251.

(5) Green, J. J.; Elisseeff, J. H. Mimicking Biological Functionality with Polymers for Biomedical Applications. Nature 2016, 386-394.

(6) Rosales, A. M.; Anseth, K. S. The Design of Reversible Hydrogels to Capture Extracellular Matrix Dynamics. Nat. Rev. Mater. 2016, 1, 15012.

(7) Hamm, P.; Zanni, M. Concepts and Methods of 2D Infrared Spectroscopy; Cambridge University Press, 2011.

(8) Kolano, C.; Helbing, J.; Kozinski, M.; Sander, W.; Hamm, P. Watching Hydrogen-Bond Dynamics in a $\beta$-Turn by Transient TwoDimensional Infrared Spectroscopy. Nature 2006, 444, 469-472.

(9) Kogan, G.; Šoltés, L.; Stern, R.; Gemeiner, P. Hyaluronic Acid: A Natural Biopolymer with a Broad Range of Biomedical and Industrial Applications. Biotechnol. Lett. 2006, 29, 17-25.

(10) Tian, X.; Azpurua, J.; Hine, C.; Vaidya, A.; Myakishev-Rempel, M.; Ablaeva, J.; Mao, Z.; Nevo, E.; Gorbunova, V.; Seluanov, A. HighMolecular-Mass Hyaluronan Mediates the Cancer Resistance of the Naked Mole Rat. Nature 2013, 499, 346-349.

(11) Singh, A.; Corvelli, M.; Unterman, S. A.; Wepasnick, K. A.; McDonnell, P.; Elisseeff, J. H. Enhanced Lubrication on Tissue and Biomaterial Surfaces through Peptide-Mediated Binding of Hyaluronic Acid. Nat. Mater. 2014, 13, 988-995.

(12) Kayitmazer, A. B.; Koksal, A. F.; Kilic Iyilik, E. Complex Coacervation of Hyaluronic Acid and Chitosan: Effects of PH, Ionic Strength, Charge Density, Chain Length and the Charge Ratio. Soft Matter 2015, 11, 8605-8612.

(13) Lalevée, G.; David, L.; Montembault, A.; Blanchard, K.; Meadows, J.; Malaise, S.; Crépet, A.; Grillo, I.; Morfin, I.; Delair, T.; et al. Highly Stretchable Hydrogels from Complex Coacervation of Natural Polyelectrolytes. Soft Matter 2017, 13, 6594-6605.

(14) Webber, M. J.; Appel, E. A.; Meijer, E. W.; Langer, R. Supramolecular Biomaterials. Nat. Mater. 2016, 15, 13-26.

(15) Rosales, A. M.; Vega, S. L.; DelRio, F. W.; Burdick, J. A.; Anseth, K. S. Hydrogels with Reversible Mechanics to Probe Dynamic Cell Microenvironments. Angew. Chem., Int. Ed. 2017, 56, 1213212136.

(16) Balazs, E. A.; Cui, J. The Story of Hyaluronan Putty. Bioact. Carbohydr. Diet. Fibre 2013, 2, 143-151.

(17) Gibbs, D. A.; Merrill, E. W.; Smith, K. A.; Balazs, E. A. Rheology of Hyaluronic Acid. Biopolymers 1968, 6, 777-791.

(18) Gatej, I.; Popa, M.; Rinaudo, M. Role of the PH on Hyaluronan Behavior in Aqueous Solution. Biomacromolecules 2005, 6, 61-67.

(19) Wu, S.; Ai, L.; Chen, J.; Kang, J.; Cui, S. W. Study of the Mechanism of Formation of Hyaluronan Putty at PH 2.5: Part I. Experimental Measurements. Carbohydr. Polym. 2013, 98, 16771682.
(20) Chakrabarti, B.; Balazs, E. A. Optical Properties of Hyaluronic Acid. Ultraviolet Circular Dichroism and Optical Rotatory Dispersion. J. Mol. Biol. 1973, 78, 135-141.

(21) Staskus, P. W.; Johnson, W. C. Conformational Transition of Hyaluronic Acid in Aqueous-Organic Solvent Monitored by Vacuum Ultraviolet Circular Dichroism. Biochemistry 1988, 27, 1522-1527.

(22) Dea, I. C.; Moorhouse, R.; Rees, D. A.; Arnott, S.; Guss, J. M.; Balazs, E. A. Hyaluronic Acid: A Novel, Double Helical Molecule. Science 1973, 179, 560-562.

(23) Sheehan, J. K.; Gardner, K. H.; Atkins, E. D. T. Hyaluronic Acid: A Double-Helical Structure in the Presence of Potassium at Low $\mathrm{PH}$ and Found Also with the Cations Ammonium, Rubidium and Caesium. J. Mol. Biol. 1977, 117, 113-135.

(24) Scott, J. E.; Heatley, F. Biological Properties of Hyaluronan in Aqueous Solution Are Controlled and Sequestered by Reversible Tertiary Structures, Defined by NMR Spectroscopy. Biomacromolecules 2002, 3, 547-553.

(25) Blundell, C. D.; Deangelis, P. L.; Almond, A. Hyaluronan: The Absence of Amide-Carboxylate Hydrogen Bonds and the Chain Conformation in Aqueous Solution Are Incompatible with Stable Secondary and Tertiary Structure Models. Biochem. J. 2006, 396, 487-498.

(26) Gribbon, P.; Heng, B. C.; Hardigham, T. E. The Analysis of Intermolecular Interactions in Concentrated Hyaluronan Solutions Suggest No Evidence for Chain-chain Association. Biochem. J. 2000, $350,329$.

(27) Mansel, B. W.; Chu, C. Y.; Leis, A.; Hemar, Y.; Chen, H. L.; Lundin, L.; Williams, M. A. K. Zooming in: Structural Investigations of Rheologically Characterized Hydrogen-Bonded Low-Methoxyl Pectin Networks. Biomacromolecules 2015, 16, 3209-3216.

(28) Selig, O.; Siffels, R.; Rezus, Y. L. A. Ultrasensitive Ultrafast Vibrational Spectroscopy Employing the Near Field of Gold Nanoantennas. Phys. Rev. Lett. 2015, 114, No. 233004.

(29) Nečas, D.; Klapetek, P. Gwyddion: An Open-Source Software for SPM Data Analysis. Cent. Eur. J. Phys. 2012, 181-188.

(30) Krężel, A.; Bal, W. A Formula for Correlating PKa Values Determined in D2O and H2O. J. Inorg. Biochem. 2004, 98, 161-166.

(31) De Marco, L.; Thämer, M.; Reppert, M.; Tokmakoff, A. Direct Observation of Intermolecular Interactions Mediated by Hydrogen Bonding. J. Chem. Phys. 2014, 141, No. 034502.

(32) Bodis, P.; Panman, M. R.; Bakker, B. H.; Mateo-Alonso, A.; Prato, M.; Buma, W. J.; Brouwer, A. M.; Kay, E. R.; Leigh, D. A.; Woutersen, S. Two-Dimensional Vibrational Spectroscopy of Rotaxane-Based Molecular Machines. Acc. Chem. Res. 2009, 42, $1462-1469$

(33) Krimm, S.; Bandekar, J. Vibrational Spectroscopy and Conformation of Peptides, Polypeptides, and Proteins. In Advances in Protein Chemistry; Academic Press, 1986; Vol. 38, pp 181-364.

(34) Woutersen, S.; Hamm, P. Isotope-edited two-dimensional vibrational spectroscopy of trialanine in aqueous solution. J. Chem. Phys. 2001, 114, 2727-2737.

(35) Hamm, P.; Lim, M.; Hochstrasser, R. M. Structure of the Amide I Band of Peptides Measured by Femtosecond NonlinearInfrared Spectroscopy. J. Phys. Chem. B 1998, 102, 6123-6138.

(36) DeCamp, M. F.; DeFlores, L.; McCracken, J. M.; Tokmakoff, A.; Kwac, K.; Cho, M. Amide I Vibrational Dynamics of NMethylacetamide in Polar Solvents: The Role of Electrostatic Interactions. J. Phys. Chem. B 2005, 109, 11016-11026.

(37) Cunha, A. V.; Salamatova, E.; Bloem, R.; Roeters, S. J.; Woutersen, S.; Pshenichnikov, M. S.; Jansen, T. L. C. Interplay between Hydrogen Bonding and Vibrational Coupling in Liquid NMethylacetamide. J. Phys. Chem. Lett. 2017, 8, 2438-2444.

(38) Cowman, M. K.; Spagnoli, C.; Kudasheva, D.; Li, M.; Dyal, A.; Kanai, S.; Balazs, E. A. Extended, Relaxed, and Condensed Conformations of Hyaluronan Observed by Atomic Force Microscopy. Biophys. J. 2005, 88, 590-602. 